\title{
Gradual Cooperation in the Existence of Outside Options*
}

by

\section{Taiji Furusawa ${ }^{\dagger}$ and Toshikazu Kawakami ${ }^{\ddagger}$}

\begin{abstract}
August 2006
Abstract

The paper shows that efficient cooperation processes exhibit gradualism when each player does not know the likelihood that the other player exercises a stochasticallyavailable outside option. Two players, asymmetrically informed on this likelihood, play an infinitely-repeated prisoner's dilemma game. Each player is either the high type with the high probability to obtain the outside option or the low type. As time proceeds with neither player exercising the outside option, each player puts more probability on the belief that his partner is the low type, enabling the players to raise cooperation levels in the efficient pooling equilibrium.
\end{abstract}

JEL Classification number: C72, C73

Keywords: Gradualism, cooperation, asymmetric information, belief updating.

*We are grateful to Takako Fujiwara-Greve, Benedetto Gui, Michihiro Kandori, seminar participants at Shinshu University, and participants of a meeting of the Japanese Economic Association, Dynamics and Information in Games and Economic Models at Keio University, and 2001 Far Eastern Meeting of the Econometric Society in Kobe for helpful comments. We acknowledge helpful comments from an anonymous referee. Furusawa gratefully acknowledges financial supports from the Seimeikai Foundation and the Ministry of Education, Culture, Sports, Science and Technology of Japan (the 21st Century Center of Excellence Project on the Normative Evaluation and Social Choice of Contemporary Economic Systems).

${ }^{\dagger}$ Corresponding author: Graduate School of Economics, Hitotsubashi University, 2-1 Naka, Kunitachi 186-8601 Japan, Email: furusawa@econ.hit-u.ac.jp, Tel/Fax: +81-42-580-8866.

${ }^{\ddagger}$ Faculty of Policy Studies, Doshisha University, Karasuma-higashi-iru, Imadegawa-dori, Kamigyou-ku Kyoto 602-8580 Japan, E-mail: tkawakam@mail.doshisha.ac.jp, Tel: +81-75-251-3478 


\section{Introduction}

In many long-term relationships, mutual cooperation "starts small" and gradually increases its level as the partnership continues. In the initial stage of cooperation, parties do not know much about each other's ability to contribute to their common interest or willingness to cooperate. As the relationship continues, this asymmetry between the parties gradually dissolves, and as a result, they increase the level of cooperation if they come to believe that the partnership is valuable.

Petersen and Rajan (1994) examine the relationships between creditors and small firms and find that the availability of financing increases with the length of the relationship. Casual observations also indicate that economic cooperation often starts small in the beginning. Credit limits of major credit cards start at low levels and then increase as customers continue to build good payment records. A manufacturer often asks a supplier to deliver only a small amount of an intermediate good when they make a fresh contract. In all such cases, parties gradually increase their mutually-beneficial transactions as they learn more about their partners in the continuing relationships. An experimental study also finds the relevance of gradualism in cooperation. Andreoni and Samuelson (2006) conduct experiments of twiceplayed prisoner's dilemma and find that as the second-period stake becomes large relative to the one in the first period, subjects are more likely to cooperate in the first period. Subjects signal their willingness to cooperate through their first-period actions and assess at the same time the opponent's willingness to cooperate.

The literature identifies causes of gradual cooperation in various settings. ${ }^{1}$ Watson (1999, 2002) shows theoretically that players gradually increase their cooperation level when they are asymmetrically informed about their willingness to cooperate. The cooperation level gradually increases so that a player who is less willing to cooperate waits until a deviation

\footnotetext{
${ }^{1}$ Furusawa and Lai (1999) show that in an infinitely repeated, prisoner's dilemma type, tariff setting game, the most-cooperative self-enforcing cooperation process exhibits gradualism in the presence of adjustment costs. Lockwood and Thomas (2002) demonstrate that if players are unable to reduce their cooperation levels, the efficient cooperation process must exhibit gradualism. Irreversibility of players' actions is also an issue in the problem of public finance. Admati and Perry (1991) and Marx and Matthews (2000) show that players' total contributions to a project are divided into smaller parts over a stretch of time. Compte and Jehiel (2004) attribute a positive correlation between a player's contribution and the other party's outside option value to gradualism in contribution games. Players gradually make contributions to prevent their respective partners from terminating the game.
} 
becomes more profitable in the future. ${ }^{2}$ Roth (1996) analyzes the situation in which players receive signals about the quality of the partnership or the value of an outside option and shows that the cooperation level evolves with the players' beliefs about those values.

In this paper, we argue that the cooperation process exhibits gradualism in the existence of stochastic outside options, if players are asymmetrically informed about their partners' likelihoods of exercising the outside option. The cooperation level evolves with the belief in these likelihoods in equilibrium. Unlike Roth, however, we study the situation in which players may be faced with different likelihoods in exercising the outside option, and these likelihoods are private information. A player who has a higher ability or lower cost of searching the outside option than the other is more likely to exercise the outside option at some point in the future. A player who is contented with his current partner is less likely to (intensively) search the outside option than the one who is discontented. It is quite natural that players are uninformed about their partners' ability, cost, or incentive to search the outside option, and hence they are usually uninformed about their partners' likelihoods of exercising the outside option. Each player, however, gradually learns about his partner's type, whether the likelihood is high or low, through a course of actions taken by his partner. Firms may collude in the product market while they compete in R\&D (Fershtman and Gandal 1994 and Martin 1995); a success in R\&D is the outside option in this case. The relationship between couples is another example. In the early stage of the relationship, couples may not be confident on each other's willingness to devote themselves into the relationship. Observing that the respective partners have not walked out of the relationship, the couples may gradually trust each other and show more devotion.

One natural type of outside options is to breach the current partnership and search for a new partner. In repeated games with random matching, Datta (1993), Ghosh and Ray (1996), Kranton (1996), Blonski and Probst (2004), and Lindsey, et al. (2003) demonstrate that the cooperation level in each partnership increases gradually. ${ }^{3}$ In the environment where

\footnotetext{
${ }^{2}$ Sobel (1985) points out the possibility that a player sends a costly message to induce the receiver to act cooperatively and then exploits him later. Watson $(1999,2002)$ extends this idea to a two-sided incomplete information game in which each player does not know whether or not his partner is willing to cooperate. Incomplete information about each other's type on the willingness to cooperate persists for a stretch of time in one equilibrium (Watson 1999), and dissolves very quickly in another (Watson 2002).

${ }^{3}$ Rob and Yang (2006) investigate a random matching model with the possibility of a continuing relationship and find a positive role of population heterogeneity (in their attitude toward cooperation) in the endogenous formation of long-term relationship. Matsushima (1990) and Fujiwara-Greve (2001) obtain the folk theorem in repeatable games with random matching.
} 
each player can change his partner immediately after he defects, players can establish a longterm relationship only if the continuation payoff from a new partnership is smaller than the continuation payoff from the current partnership. This condition can only be satisfied when players are supposed to start a new partnership with a low cooperation level.

We analyze the situation in which two players, asymmetrically informed on the likelihood that the other player exercises an outside option, play an infinitely-repeated prisoner's dilemma game. ${ }^{4}$ There are two types of player that we call high and low. The high type has a higher probability to obtain the outside option than the low type. Indeed, to simplify the exposition, we assume that the low type does not have access to the outside option (i.e., the probability for the low type to obtain the outside option is zero). We suppose, on the other hand, that the value of the outside option is so large that the high type exercises the option whenever it becomes available. A drastic R\&D allows the successful firm to enjoy monopoly profits, greater than a share of pre-innovation collusive profits. A person who is discontented with the current partner switches to a new partner only if her new partner is closer to her ideal type.

Section 3 analyzes the pooling equilibrium. As time proceeds with neither player exercising the outside option, each player puts more probability on the belief that his partner is the low type, raising the subjective probability of the event that the partnership lasts perpetually. The expected value of cooperation increases as the game progresses, enabling the players to raise cooperation levels in the efficient pooling equilibrium. ${ }^{5}$ In Section 4 , we derive separating equilibria, in which the players' first period actions reveal their types. Players "test" their respective partners by setting an "experimental" level of cooperation in the first period, and if both (or at least one, in some cases) players pass the "test," they choose the maximal cooperation level at a stretch from the second period onward. ${ }^{6}$

Then, we compare the efficiency between the pooling and separating equilibria. In the situation where it is relatively hard for players to cooperate (e.g., players are impatient), the separating equilibrium tends to be more efficient since even in the case where no cooperation

\footnotetext{
${ }^{4}$ Fudenberg and Maskin (1986) establish the folk theorem for finitely-repeated games with incomplete information such that whether or not a player is "sane" or "crazy" is private information. We focus on the efficient equilibrium (rather than the entire set of equilibrium) under particular information incompleteness regarding the likelihood of exercising the outside option.

${ }^{5}$ The dynamic process of the efficient pooling equilibrium is similar to the one that Watson (1999) obtains.

${ }^{6}$ The dynamic process of our separating equilibrium is similar to the ones that Ghosh and Ray (1996), Kranton (1996), and Watson (2002) obtain.
} 
is sustainable in the pooling equilibrium, the full cooperation can be sustained from the second period when both players are revealed to be the low type. In the case where it is relatively easy for players to cooperate, on the other hand, the pooling equilibrium tends to be more efficient, since full cooperation is likely to be achieved from the beginning in the pooling equilibrium whereas in the separating equilibrium there is always a chance that cooperation will not be achieved.

We show in Section 4 that as the asymmetry between the two types of player in the likelihood of exercising the outside option increases, cooperation becomes more difficult to achieve in the pooling equilibrium, and hence the separating equilibrium tends to be more efficient. In a framework of a complete-information repeated game, Bardhan (2004, Chapter 10) examines how the disparity in their private capital holdings affects the possibility of cooperation between two agents in their efforts of increasing capital productivity. He demonstrates that cooperation cannot be achieved if the distributional inequality exceeds a certain level; the poor agent has strong incentive to appropriate all the fruits of cooperation in such cases. The difference in capital holdings entails differential exit options between agents, as well as differential benefits from cooperation. Bardhan argues that this difference in exit options is important in the problem of managing a common pool of resources in rural areas of developing countries. The difference in exit options is important not only in the common pool problem in rural areas of developing countries, but also in many socioeconomic relationships in which partners' exit options are usually not as visual as in the common pool problem in rural areas. The model framework of this paper enables us to assess how the asymmetry in exit options affects cooperation when exit options are private information. One of our findings is that as the asymmetry in the privately-informed likelihood of exercising the outside option increases, agents are less likely to build the relationship gradually (the pooling equilibrium), but more likely to judge very quickly whether or not they should continue the relationship (the separating equilibrium).

\section{The Model}

We consider an infinitely-repeated game with stochastic outside options, which is played by ex ante symmetric two players who discount the future with the common discount factor $\delta \in(0,1)$. In any period $t=1,2, \cdots$, each player $i \in I \equiv\{1,2\}$ chooses his own cooperation level, $\pi^{i}(t) \in[0, \bar{\pi}]$. The stage game has the structure of the prisoner's dilemma: a player's 
payoff is higher if the other player's cooperation level is higher or his own cooperation level is lower. We consider a simple stage-game payoff function, $u:[0, \bar{\pi}]^{2} \rightarrow \mathbf{R}$, which captures these features; player $i$ 's payoff is given by

$$
u\left(\pi^{i}(t), \pi^{-i}(t)\right)=\beta \pi^{-i}(t)-(\beta-1) \pi^{i}(t),
$$

where $\beta>1$ and $-i$ represents player $i$ 's partner. When both players select the same cooperation level $\pi$, they individually obtain $u(\pi, \pi)=\pi$. When player $i$ chooses his dominant strategy $\pi^{i}(t)=0$ while his partner chooses $\pi^{-i}(t)=\pi$, player $i$ obtains $u(0, \pi)=\beta \pi$ while player $-i$ obtains $u(\pi, 0)=-(\beta-1) \pi$. It is clear that this stage game has a unique Nash equilibrium $\left(\pi^{1}(t), \pi^{2}(t)\right)=(0,0)$, in which they individually obtain $u(0,0)=0$. Notice that if they cooperatively select a higher $\pi$, they can enjoy a higher payoff. However, the payoff from deviation is also high when the cooperation level is high.

There are two types of player, low and high, differing in the likelihood of exercising the outside option. It is common knowledge that in the beginning of the game, each player is either the low type with probability $p_{1}$ or the high type with probability $1-p_{1}$. The actual type of each player, however, is private information. The high type has access to the outside option of the value $v$, which arrives with probability $q \in(0,1)$ in each period, whereas the low type has no access. We assume for simplicity that outside options are independent between the players. Thus, the high type continues to have stochastic access to the outside option even after his rival has exercised the outside option. ${ }^{7}$ Moreover, even if both players simultaneously exercise the outside option, each player obtains the full value $v$. Throughout the analysis, we assume $v \geq \bar{\pi} /(1-\delta)$, that is, the value of the outside option is greater than or equal to the present value of perpetual full cooperation. Under this assumption, the high type always exercises the outside option when it becomes available rather than continuing cooperation with his partner. ${ }^{8}$

In every period but the first, whether or not the outside option is available is revealed to high-type players before the two players choose their individual cooperation levels. ${ }^{9}$ It

\footnotetext{
${ }^{7}$ In the example of semi-cooperation between two firms (colluding in the product market while competing in $R \& D)$, this assumption corresponds to the case in which the high type continues to engage in $R \& D$ activities even after the other firm succeeded in a drastic innovation.

${ }^{8}$ It can be shown that if this assumption is violated, players fully cooperate from the beginning without seeking the outside option when the incentive to defect is not so large. With this assumption, we can concentrate on the case where the equilibrium exhibits gradualism and unveil the mechanism through which the existence of outside options causes gradual cooperation. Interested readers are invited to the Concluding Remarks of Furusawa and Kawakami (2006) for further discussions on this assumption.

${ }^{9}$ We assume that there is no chance for a high type player to obtain the outside option in period 1 to avoid
} 
is revealed publicly whether or not each player has exercised the outside option. If at least one player exercises the outside option, he (and only he) receives the payoff $v$ and the game ends. If neither player exercises the outside option, the two players simultaneously select their individual cooperation levels and receive their stage-game payoffs. Since the arrival of the outside option ends the game, the history of the game can be expressed simply by the past cooperation profiles. Let $\pi(t) \equiv\left(\pi^{1}(t), \pi^{2}(t)\right)$ denote the cooperation profile in period $t$, and let $h_{1}$ denote the empty history and $h_{t}=(\pi(1), \cdots, \pi(t-1))$, for $t \geq 2$, the history up to period $t$ (after it is revealed that neither player exercises the outside option in period t). Also let $H_{t}$ be the set of all $h_{t}$, and $H \equiv \cup_{t \in \mathbf{N}} H_{t}$, where $\mathbf{N}$ stands for the set of positive integers. Player $i$ 's type $\theta^{i}$ is an element of the set $\Theta=\{l, h\}$, where $l$ and $h$ signify the low and high types, respectively. Player $i$ 's strategy is a function $\sigma^{i}: H \times \Theta \rightarrow[0, \bar{\pi}]$, and player $i$ 's belief that his partner is the low type is a function $\mu^{i}: H \times \Theta \rightarrow[0,1]$.

We say that an equilibrium outcome path $\{\pi(t)\}_{t=1}^{\infty}$, or equilibrium in short, exhibits gradualism if, for any $i=1,2, \pi^{i}(t+1) \geq \pi^{i}(t)$ for every $t \in \mathbf{N}$ with strict inequality for some $t$. We show in the following that the symmetric, efficient, perfect Bayesian, pooling equilibrium exhibits gradualism.

\section{Pooling Equilibrium}

Our model admits two types of equilibrium, pooling and separating. The first and main part of the paper focuses on the pooling equilibrium in which neither player reveals his own type until the outside option possibly arrives.

\subsection{The Efficient Cooperation Path}

We derive a symmetric, efficient, self-enforcing, perfect Bayesian equilibrium. In each period, the two players select the maximal cooperation level that satisfies their individual incentive constraints. They continue to cooperate until either a player exercises the outside option or a player defects. We assume that if the cooperation ends with a defection, they continue to engage in the stage-game Nash equilibrium, $\left(\pi^{1}(t), \pi^{2}(t)\right)=(0,0)$, from the next period onwards. Notice that this Nash reversion is the harshest punishment since each player

a superfluous outcome in which the game ends before the players choose their first individual cooperation levels. 
receives his minimax payoff in every period.

Let $\left\{\pi_{t}^{c}\right\}_{t=1}^{\infty}$ and $\left\{p_{t}\right\}_{t=1}^{\infty}$ denote the cooperation and belief sequences, respectively. In each period $t$, both players choose a common cooperation level $\pi_{t}^{c}$ (i.e., $\pi(t)=\left(\pi_{t}^{c}, \pi_{t}^{c}\right)$ ), such that $\pi_{t}^{c}$ is the maximal cooperation level that satisfies the incentive constraint for either type. Let $\mathcal{P}(I)$ denote the set of all subsets of $I$. Then, we define by $H_{t}^{A, s}$ for $t \geq 2$ the set of all histories up to period $t$ in which the first deviation from the cooperation sequence (by setting a cooperation level lower than $\pi_{s}^{c}$ ) occurs in period $s \leq t-1$ by the set of players $A \in \mathcal{P}(I)$. When neither player has deviated by period $t$, the date of deviation $s$ is irrelevant, and hence we simply write $H_{t}^{\emptyset}$ to represent the set of such histories. To simplify the exposition, we define $H_{1}^{\emptyset}$ to be the same as $H_{1}$, the set that consists only of the empty history $h_{1}$. Then, player $i$ 's strategy is given by

$$
\sigma^{i}\left(h_{t}, \theta^{i}\right)=\left\{\begin{array}{cl}
\pi_{t}^{c} & \text { if } h_{t} \in H_{t}^{\emptyset} \\
0 & \text { otherwise }
\end{array}\right.
$$

which does not depend on his type.

In the course of cooperation, the players update their individual beliefs about the other player's type. At the beginning of period $t$, they attach the same probability $p_{t}$ to the event that the other player is the low type, due to the symmetry of the model. Player $i$ 's belief in period $t$ that his partner is the low type is given by $\mu^{i}\left(h_{t}, \theta^{i}\right)=p_{t}$ for any $h_{t} \in H_{t}$ and for any $\theta^{i} \in \Theta .{ }^{10}$ The subjective probability $p_{t}$ evolves according to the Bayes rule. Each player updates his belief such that

$$
p_{t+1}=p_{t} /\left[p_{t}+\left(1-p_{t}\right)(1-q)\right]
$$

if his partner has not exercised the outside option at the beginning of period $t+1$. Repeated application of (1) yields $p_{t}=p_{1} /\left[p_{1}+\left(1-p_{1}\right)(1-q)^{t-1}\right]$, which gives us the belief sequence $\left\{p_{t}\right\}_{t=1}^{\infty}$ and the following lemma.

Lemma 1 As $t$ increases, $p_{t}$ increases to 1.

\footnotetext{
${ }^{10}$ We assume here that players' beliefs about the other player's type do not depend on their past actions. Alternatively, we can specify the out-of-equilibrium belief such that a player comes to believe that his partner is the high type, for example, when observing his partner's deviation. However, since a deviation entails the punishment phase, in which both players continue to select zero cooperation level regardless of the type of player, the selection of the out-of-equilibrium beliefs would not affect the equilibrium outcome. Thus, our equilibrium selection with regard to the out-of-equilibrium beliefs is innocuous.
} 
The belief that the other player is the low type increases as time proceeds as long as neither player exercises the outside option.

Conditional on the information available in period $t$, the probability that a player believes the other player stays in the cooperation arena in period $t+1$ is the sum of the probability that the player believes the other player is the low type and the joint probability that the player believes the other player is the high type but fails in obtaining the outside option at the beginning of period $t+1$. Let

$$
P_{t+1} \equiv p_{t}+\left(1-p_{t}\right)(1-q)
$$

denote this subjective probability. Then the Bayes formula (1) can be rewritten as $p_{t+1}=$ $p_{t} / P_{t+1}$. Repeated application of this relationship and (2) gives us $P_{t+s}=\left[p_{t}+\left(1-p_{t}\right)(1-\right.$ $\left.q)^{s}\right] / \prod_{j=1}^{s-1} P_{t+j}$, or equivalently

$$
\prod_{j=1}^{s} P_{t+j}=p_{t}+\left(1-p_{t}\right)(1-q)^{s}
$$

which represents the conditional subjective probability in period $t$ that the partner stays in the cooperation arena in period $t+s$.

Now, let us derive the maximal cooperation path $\left\{\pi_{t}^{c}\right\}_{t=1}^{\infty}$, such that the strategy profile and belief system characterized in the above constitute a perfect Bayesian equilibrium.

First, we consider the incentive constraint for the low type. Since the low type will not exercise the outside option, his subjective probability that both players stay in the cooperation arena in period $t+s$ conditional on the information available in period $t$ is $\prod_{j=1}^{s} P_{t+j}$. Thus, the present discounted payoff (as of period $t$ ) from conforming to the equilibrium cooperation path equals $\pi_{t}^{c}+\sum_{s=1}^{\infty} \delta^{s} \prod_{j=1}^{s} P_{t+j} \pi_{t+s}^{c}$. On the other hand, the present discounted payoff from deviation equals $\beta \pi_{t}^{c}$. Consequently, the incentive constraint for the low type in period $t$ can be written as

$$
(\beta-1) \pi_{t}^{c} \leq \sum_{s=1}^{\infty} \delta^{s} \prod_{j=1}^{s} P_{t+j} \pi_{t+s}^{c} .
$$

The high type is faced with a similar incentive constraint except that he takes into account the possibility of exercising the outside option. In equilibrium, the high type continues to expect a flow of future payoffs from cooperation until either player exercises the outside option. The present discounted payoff from cooperation in period $t, V_{t}$, can be written as

$$
V_{t}=\pi_{t}^{c}+\delta\left[q v+(1-q)\left[P_{t+1} \pi_{t+1}^{c}+\delta\left[q v+(1-q)\left[P_{t+1} P_{t+2} \pi_{t+2}^{c}+\cdots\right.\right.\right.\right.
$$




$$
=\pi_{t}^{c}+\sum_{s=1}^{\infty} \delta^{s}(1-q)^{s} \prod_{j=1}^{s} P_{t+j} \pi_{t+s}^{c}+\sum_{s=1}^{\infty} \delta^{s} q(1-q)^{s-1} v .
$$

Since the probability to exercise the outside option does not change even if the players' mutual cooperation ceases, the present discounted payoff from defection $V_{t}^{d}$ is given by

$$
V_{t}^{d}=\beta \pi_{t}^{c}+\sum_{s=1}^{\infty} \delta^{s} q(1-q)^{s-1} v
$$

Thus, the incentive constraint for the high type in period $t$ can be written as

$$
(\beta-1) \pi_{t}^{c} \leq \sum_{s=1}^{\infty} \delta^{s}(1-q)^{s} \prod_{j=1}^{s} P_{t+j} \pi_{t+s}^{c}
$$

Comparing (4) and (5), we immediately realize that (5) is tighter than (4). The high type's subjective probability that both players continue to be in the cooperation arena is lower than the low type's, since the high type has private information that he may leave the cooperation arena sometime soon, making the expected loss from breaking cooperation smaller. The efficient, self-enforcing cooperation sequence consists of the maximal $\pi_{t}^{c}$ that satisfies (5) for every $t \geq 1$. Substituting (3) into (5) gives us the reduced form of the incentive constraint that must be satisfied in equilibrium:

$$
(\beta-1) \pi_{t}^{c} \leq \sum_{s=1}^{\infty} \delta^{s}(1-q)^{s}\left[p_{t}+\left(1-p_{t}\right)(1-q)^{s}\right] \pi_{t+s}^{c}
$$

Now, let us derive the condition under which full cooperation can be sustained perpetually once it is attained. We substitute $\bar{\pi}$ for $\pi_{t+s}^{c}$ for every $s \geq 0$ in (6) to obtain

$$
(\beta-1) \bar{\pi} \leq \sum_{s=1}^{\infty} \delta^{s}(1-q)^{s}\left[p_{t}+\left(1-p_{t}\right)(1-q)^{s}\right] \bar{\pi},
$$

which can be reduced to

$$
p_{t} \geq \hat{p}
$$

where

$$
\hat{p} \equiv \frac{[1-\delta(1-q)]\left[\beta-1-\beta \delta(1-q)^{2}\right]}{\delta q(1-q)} .
$$

It follows from Lemma 1 that if (8) is satisfied in period $t$, full cooperation is sustained thereafter until a player possibly exercises the outside option.

Lemma 2 If $p_{t} \geq \hat{p}$, full cooperation is sustained from period $t$ onward until a player possibly exercises the outside option. 
It is readily verified from (9) that $\hat{p}>0$ if and only if $(\beta-1) / \beta>\delta(1-q)^{2}$, while $\hat{p}<1$ if and only if $(\beta-1) / \beta<\delta(1-q)$. Since (8) always holds for any $p_{t}(t \geq 1)$ if $\hat{p} \leq 0$, we have the following.

Proposition 1 If $(\beta-1) / \beta \leq \delta(1-q)^{2}$, full cooperation is attained immediately and sustained thereafter until a player exercises the outside option.

The high type's subjective probability that both players will be in the cooperation arena in the next period is either $1-q$ if he holds the optimistic belief that his partner is the low type or $(1-q)^{2}$ if he holds the pessimistic belief that his partner is the high type. He is willing to cooperate if the attractiveness to defect, represented by $(\beta-1) / \beta$, does not exceed the lowest effective discount factor, $\delta(1-q)^{2}$, which corresponds to the pessimistic belief. Immediate full cooperation is more likely to be realized if (i) the attractiveness to defect is small (i.e., $(\beta-1) / \beta$, or $\beta$, is small), (ii) the two players are patient (i.e., $\delta$ is large), and (iii) the probability that the high type obtains the outside option is small (i.e., $q$ is small).

Next, we turn to the case where $\delta(1-q)^{2}<(\beta-1) / \beta<\delta(1-q)$, that is, $0<\hat{p}<1$. Letting $\hat{t} \equiv \min \left\{t: p_{t} \geq \hat{p}\right\}$, we immediately obtain the following from Lemma 1 and Lemma 2 .

Lemma 3 When $\delta(1-q)^{2}<(\beta-1) / \beta<\delta(1-q)$, the two players eventually enter the full cooperation phase (i.e., $\pi_{t}^{c}=\bar{\pi}$ for all $t \geq \hat{t}$ ), unless a player exercises the outside option by $\hat{t}$.

If $p_{1} \geq \hat{p}$, the two players fully cooperate from period 1 . If $p_{1}<\hat{p}$, however, they cannot fully cooperate in the beginning. They must start small and gradually raise the cooperation level until period $\hat{t}$, as we show below.

The maximal cooperation level in period $\hat{t}-1$ must satisfy the high type's incentive constraint with equality. The belief $p_{\hat{t}-1}$ is smaller than $\hat{p}$, but large enough that the belief in the next period exceeds $\hat{p}$ (i.e., $p_{\hat{t}} \geq \hat{p}$ ). It is convenient to introduce the lag operator $L$ with which the Bayes formula (1) is simply expressed by $p_{t}=L\left(p_{t+1}\right)$, that is, $p_{t+1} \equiv$ $L\left(p_{t+1}\right) /\left[L\left(p_{t+1}\right)+\left(1-L\left(p_{t+1}\right)\right)(1-q)\right]$. Then, it follows from $(6)$ that for $p_{\hat{t}-1} \in[L(\hat{p}), \hat{p})$, the relevant incentive constraint is

$$
(\beta-1) \pi_{\hat{t}-1}^{c}=\sum_{s=1}^{\infty} \delta^{s}(1-q)^{s}\left[p_{\hat{t}-1}+\left(1-p_{\hat{t}-1}\right)(1-q)^{s}\right] \bar{\pi} .
$$


The right-hand side increases linearly with $p_{\hat{t}-1}$, and so does $\pi_{\hat{t}-1}^{c}$. Since (7) and (8) imply that the right-hand side of (10) equals $(\beta-1) \bar{\pi}$ if $p_{\hat{t}-1}=\hat{p}$, we find that $\pi_{\hat{t}-1}^{c}<\bar{\pi}$ as $p_{\hat{t}-1}<\hat{p}$. Thus, we have shown the following lemma.

Lemma 4 Consider the case where $\delta(1-q)^{2}<(\beta-1) / \beta<\delta(1-q)$. In period $\hat{t}-1$, when $p_{\hat{t}-1} \in[L(\hat{p}), \hat{p})$, each player's cooperation level equals $\pi_{\hat{t}-1}^{c}=\sum_{s=1}^{\infty} \delta^{s}(1-q)^{s}\left[p_{\hat{t}-1}+(1-\right.$ $\left.\left.p_{\hat{t}-1}\right)(1-q)^{s}\right] \bar{\pi} /(\beta-1)$, which is smaller than $\bar{\pi}$.

Figure 1 shows the maximal cooperation process; the upper diagram shows the maximal cooperation level as a function of the belief, while the lower diagram shows how the belief is updated. Thus far, we have derived the schedule in the lower diagram and the part of the schedule corresponding to $[L(\hat{p}), 1]$ in the upper diagram.

We derive the rest of the schedule by backward induction. Suppose for $t \leq \hat{t}-2$ that we have derived the maximal cooperation schedule corresponding to period $t+1$ onward (i.e., we have derived the schedule for $\left.\left[L^{\hat{t}-t-1}(\hat{p}), 1\right]\right)$. Then, for $p_{t} \in\left[L^{\hat{t}-t}(\hat{p}), L^{\hat{t}-t-1}(\hat{p})\right)$, we derive $\pi_{t}^{c}$ that satisfies the incentive constraint in period $t$ with equality:

$$
(\beta-1) \pi_{t}^{c}=\sum_{s=1}^{\infty} \delta^{s}(1-q)^{s}\left[p_{t}+\left(1-p_{t}\right)(1-q)^{s}\right] \pi_{t+s}^{c}
$$

We simplify (11) using the fact that the incentive constraint is also binding in period $t+1$. The binding incentive constraint in period $t+1$ can be written as

$$
(\beta-1) \pi_{t+1}^{c}=\sum_{s=2}^{\infty} \delta^{s-1}(1-q)^{s-1}\left[p_{t+1}+\left(1-p_{t+1}\right)(1-q)^{s-1}\right] \pi_{t+s}^{c} .
$$

To derive the relationship between $\pi_{t}^{c}$ and $\pi_{t+1}^{c}$ from the above two equalities, we notice the following relationship derived from (3): For any $s \geq 1$ and $0 \leq k \leq s$,

$$
\begin{aligned}
p_{t}+ & \left(1-p_{t}\right)(1-q)^{s}=\prod_{j=1}^{s} P_{t+j}=\prod_{j=1}^{k} P_{t+j} \Pi_{j=1}^{s-k} P_{t+k+j} \\
& =\left[p_{t}+\left(1-p_{t}\right)(1-q)^{k}\right]\left[p_{t+k}+\left(1-p_{t+k}\right)(1-q)^{s-k}\right] .
\end{aligned}
$$

The event that the other players will not exercise the outside option until period $t+s$ is equivalent to the joint event that he will not exercise it until $t+k$ and that he will continue to fail in obtaining the outside option for the rest of $s-k$ periods. The relationship (13) reflects this equivalence. 
Taking account of (13) when $k=1$ and multiplying both sides by $\delta(1-q)$, we reduce (12) to

$$
\delta(\beta-1)(1-q)\left[p_{t}+\left(1-p_{t}\right)(1-q)\right] \pi_{t+1}^{c}=\sum_{s=2}^{\infty} \delta^{s}(1-q)^{s}\left[p_{t}+\left(1-p_{t}\right)(1-q)^{s}\right] \pi_{t+s}^{c} .
$$

Substituting this equality into (11), we obtain

$$
\pi_{t}^{c}=\left(\frac{\beta}{\beta-1}\right) \delta(1-q)\left[p_{t}+\left(1-p_{t}\right)(1-q)\right] \pi_{t+1}^{c} .
$$

It is readily verified (in the proof of Lemma 5, relegated to the Appendix) that the coefficient of $\pi_{t+1}^{c}$ in equality (14) is strictly less than 1 , so $\pi_{t}^{c}$ is strictly smaller than $\pi_{t+1}^{c} \cdot{ }^{11}$

Lemma 5 In period $t=1, \cdots, \hat{t}-2, \pi_{t}^{c}$ is determined by

$$
\pi_{t}^{c}=\left(\frac{\beta}{\beta-1}\right) \delta(1-q)\left[p_{t}+\left(1-p_{t}\right)(1-q)\right] \pi_{t+1}^{c}
$$

for a given $\pi_{t+1}^{c}$. The coefficient $\beta \delta(1-q)\left[p_{t}+\left(1-p_{t}\right)(1-q)\right] /(\beta-1)$ is strictly less than 1 for any $p_{t}<\hat{p}$ when $0<\hat{p}<1$.

From Lemma 3, Lemma 4, and Lemma 5, we can complete the maximal cooperation schedule as Figure 1 depicts. Figure 1 also shows a cooperation process when $p_{1}$ lies between $L^{2}(\hat{p})$ and $L(\hat{p})$, in which the cooperation level gradually increases from $\pi_{1}$ to $\bar{\pi}$ in three periods as the belief is updated.

Given the threshold beliefs $\left\{L^{s}(\hat{p})\right\}_{s=0}^{\infty}$, which can be calculated from (1) and (9), we can further proceed to find the expression that characterizes the maximal cooperation level as a function of the belief. To this end, we first apply (14) repeatedly, using (13), to obtain the relationship between $\pi_{t}^{c}$ and $\pi_{\hat{t}-1}^{c}$ for $t<\hat{t}-1$ :

$$
\pi_{t}^{c}=\left[\left(\frac{\beta}{\beta-1}\right) \delta(1-q)\right]^{\hat{t}-t-1}\left[p_{t}+\left(1-p_{t}\right)(1-q)^{\hat{t}-t-1}\right] \pi_{\hat{t}-1}^{c} .
$$

Then, we substitute the expression derived in Lemma 4 for $\pi_{\hat{t}-1}^{c}$ in the above equation to obtain

$$
\pi_{t}^{c}=\left[\left(\frac{\beta}{\beta-1}\right) \delta(1-q)\right]^{\hat{t}-t-1}\left[p_{t}+\left(1-p_{t}\right)(1-q)^{\hat{t}-t-1}\right]
$$

\footnotetext{
${ }^{11}$ The Appendix is available on the website of the Journal of Economic Behavior and Organization.
} 


$$
\begin{aligned}
& \times \frac{1}{\beta-1} \sum_{s=1}^{\infty} \delta^{s}(1-q)^{s}\left[p_{\hat{t}-1}+\left(1-p_{\hat{t}-1}\right)(1-q)^{s}\right] \bar{\pi} \\
= & \frac{1}{\beta}\left[\left(\frac{\beta}{\beta-1}\right) \delta(1-q)\right]^{\hat{t}-t} \sum_{s=1}^{\infty} \delta^{s-1}(1-q)^{s-1}\left[p_{t}+\left(1-p_{t}\right)(1-q)^{\hat{t}-t+s-1}\right] \bar{\pi} \\
= & \frac{1}{\beta}\left[\left(\frac{\beta}{\beta-1}\right) \delta(1-q)\right]^{\hat{t}-t} \sum_{s=0}^{\infty} \delta^{s}(1-q)^{s}\left[p_{t}+\left(1-p_{t}\right)(1-q)^{\hat{t}-t+s}\right] \bar{\pi},
\end{aligned}
$$

where we have used (13) in the derivation. The expression can further be reduced to

$$
\pi_{t}^{c}=\frac{1}{\beta}\left(\frac{\beta}{\beta-1}\right)^{\hat{t}-t}\left[\frac{\delta^{\hat{t}-t}(1-q)^{\hat{t}-t}}{1-\delta(1-q)} p_{t}+\frac{\delta^{\hat{t}-t}(1-q)^{2(\hat{t}-t)}}{1-\delta(1-q)^{2}}\left(1-p_{t}\right)\right] \bar{\pi},
$$

which indicates that the maximal cooperation schedule is piecewise linear where kinks occur at $\left\{L^{s}(\hat{p})\right\}_{s=0}^{\infty}$. The following proposition summarizes the discussion.

Proposition 2 When $\delta(1-q)^{2}<(\beta-1) / \beta<\delta(1-q)$, if the initial belief that the other player is the low type is small such that $p_{1}<\hat{p}$, then the efficient, self-enforcing cooperation level increases gradually (i.e., the equilibrium cooperation path exhibits gradualism).

Next, let us consider the case where $(\beta-1) / \beta=\delta(1-q)$, i.e., $\hat{p}=1$. The incentive constraint (7) implies that as $p_{t}$ increases to 1 , the cooperation level approaches $\bar{\pi}$ although it never reaches $\bar{\pi}$. Indeed, the cooperation process is very simple, such that the maximal cooperation level $\pi_{t}^{c}$ equals $p_{t} \bar{\pi}$.

Proposition 3 When $(\beta-1) / \beta=\delta(1-q)$, the maximal, self-enforcing cooperation level is given by $\pi_{t}^{c}=p_{t} \bar{\pi}$. As the belief is updated, the cooperation level increases gradually and approaches $\bar{\pi}$.

The proof of this proposition is relegated to the Appendix.

Finally, if $(\beta-1) / \beta>\delta(1-q)$, that is, $\hat{p}>1$, the attractiveness to defect exceeds the high type's effective discount factor that corresponds to the optimistic belief. In this case, the attractiveness to defect is so large, the players are so impatient, or the probability that the high type obtains the outside option is so large that no cooperation takes place in any period. This result is intuitive, so the proof of the following proposition is omitted. ${ }^{12}$

Proposition 4 When $(\beta-1) / \beta>\delta(1-q)$, no cooperation takes place in any period.

\footnotetext{
${ }^{12}$ Interested readers are invited to Furusawa and Kawakami for the proof of Proposition 4.
} 


\subsection{Comparative Dynamics}

Proposition 2 shows that if the attractiveness to defect is in the intermediate range and the initial belief that the partner is the low type is small, the maximal, self-enforcing cooperation path exhibits gradualism. We show here that how this gradual cooperation process is affected by a change in some parameter values of the model: (i) the attractiveness to defect $\beta$, (ii) the players' discount factor $\delta$, and (iii) the arrival rate of the outside option $q$.

It follows immediately from (1) that the belief updating process is not affected by a change in $\beta$ or $\delta$, whereas a change in $\beta$ or $\delta$ affects the maximal cooperation schedule. We find from (9) and (16) that as $\beta$ rises or $\delta$ falls, the maximal cooperation schedule, depicted in the upper diagram of Figure 1, shifts down.

Proposition 5 As the attractiveness to defect increases or the players become less patient, the cooperation level in each period becomes lower, and it takes weakly longer for the cooperation level to reach its maximum. The present discounted payoffs from cooperation also become lower as a result.

As $\beta$ becomes higher or $\delta$ becomes lower, the incentive to deviate increases relative to the incentive to keep cooperation. To prevent a deviation, the players must be content with lower cooperation levels in the "trust-building" phase.

An increase in $q$, the probability that the high type obtains the outside option, affects the cooperation path through two channels. First, it accelerates the belief updating process, as (1) indicates, since the news that the other player has not exercised the outside option makes a player more confident that his partner is the low type. This effect is reflected as a downward shift of the belief updating schedule in the lower diagram of Figure 1. Second, it shifts down the maximal cooperation schedule as (9) and (15) indicate. A simple but somewhat tedious computation, which is relegated to the Appendix, shows that $\hat{p}$ increases with $q$ when $0<\hat{p}<1$. The equation (15) shows that $\pi_{t}^{c}$ decreases with $q$ for a given $p_{t}$; an increase in $q$ reduces the future benefits from cooperation so that the cooperation level must be reduced to make a deviation less attractive.

Although an increase in $q$ decreases the cooperation level for any level of the belief, the belief that the partner is the low type itself increases more quickly. Therefore, an increase in $q$ may enable the players to realize a higher cooperation level at some point in the cooperation path, which makes the impact on the present discounted payoffs ambiguous. 
Proposition 6 As the probability that the high type obtains the outside option increases, the belief that his partner is the low type increases more quickly while the cooperation level that they can attain for each level of the belief decreases. Whether or not the cooperation level in each period increases depends on which effect outweighs the other.

\section{Separating Equilibrium}

We have derived the efficient pooling equilibrium in which a player's type is revealed only when the outside option is exercised. In this section, we derive separating equilibria in which each player's type is revealed through his first-period action. Our results here are counterparts of the results by Ghosh and Ray (1996), Blonski and Probst (2004), and Watson (2002) who show in their respective models that two players, starting with a low cooperation level, learn each other's types very quickly. We show in our model that separating equilibria exist in a wider parameter range than pooling equilibria.

If the attractiveness to defect is so large that $(\beta-1) / \beta>\delta$, we have $\beta \pi>\pi /(1-\delta)$ for any $\pi \in(0, \bar{\pi}]$, and hence the two players cannot sustain cooperation even when both of them are revealed to be the low type. The only perfect Bayesian equilibrium outcome in this case is the repetition of the stage-game Nash equilibrium. If the attractiveness to defect is small so that $(\beta-1) / \beta<\delta(1-q)^{2}$, on the other hand, the players can attain full cooperation immediately and sustain it thereafter in the pooling equilibrium (Proposition 1). Even though there also exist separating equilibria in this case, all such equilibria must involve partial cooperation from the second period when players have been revealed to be the high type in the first period. ${ }^{13}$ Since the players can sustain full cooperation even when both players are revealed to be the high type in this case, these separating equilibria are inefficient and somewhat artificial. Therefore, we will not pursue this derivation, but rather focus on the case where the attractiveness to defect is in the intermediate range: $\delta(1-q)^{2}<(\beta-1) / \beta \leq \delta$.

We begin with the case where the attractiveness to defect is relatively large such that $\delta(1-q)<(\beta-1) / \beta \leq \delta$. In equilibrium, the low type selects a possibly low cooperation level in period 1 and then offers full cooperation in the following periods if and only if he comes to believe that his partner is also the low type, whereas the high type never cooperates. To

\footnotetext{
${ }^{13}$ If the players fully cooperate from the second period onwards regardless of their first-period actions, each player's rational action in the first period is to select his dominant strategy of choosing 0 . Thus, any separating equilibrium must involve partial cooperation from the second period.
} 
describe the equilibrium strategy profile, we first select a cooperation sequence $\left\{\pi_{t}^{c}\right\}_{t=1}^{\infty}$ such that $\pi_{1}^{c}$ satisfies

$$
\frac{p_{1} \delta(1-q) \beta \bar{\pi}}{\beta-1} \leq \pi_{1}^{c} \leq \frac{p_{1} \delta \bar{\pi}}{(1-\delta)(\beta-1)},
$$

and $\pi_{t}^{c}=\bar{\pi}$ for any $t \geq 2$. Then player $i$ 's strategy in any period $t$ is given by

$$
\begin{aligned}
& \sigma^{i}\left(h_{t}, l\right)=\left\{\begin{array}{cc}
\pi_{t}^{c} & \text { if } h_{t} \in H_{t}^{\emptyset} \\
0 & \text { otherwise }
\end{array}\right. \\
& \sigma^{i}\left(h_{t}, h\right)=0 \text { for any } h_{t} \in H_{t},
\end{aligned}
$$

while his belief that his partner is the low type is given by

$$
\mu^{i}\left(h_{t}, \theta^{i}\right)=\left\{\begin{array}{cl}
p_{1} & \text { if } h_{t} \in H_{1} \\
0 & \text { if } h_{t} \in H_{t}^{\{-i\}, 1} \cup H_{t}^{I, 1} \\
1 & \text { otherwise }
\end{array}\right.
$$

for any $\theta^{i}$. Player $i$ believes that his partner is the high type if his partner has deviated from selecting $\pi_{1}^{c}$ in the first period, but otherwise he believes that his partner is the low type. He maintains this belief throughout the game. ${ }^{14}$ The following proposition, whose proof is relegated to the Appendix, claims that the strategy profile and the system of belief described in the above constitute a perfect Bayesian equilibrium.

Proposition 7 When $\delta(1-q)<(\beta-1) / \beta \leq \delta$, there exists a separating equilibrium in which full cooperation is sustained from the second period onward if and only if both players are revealed to be the low type through their actions in the first period.

When $\delta(1-q)<(\beta-1) / \beta$, the players cannot sustain cooperation if either of them is revealed to be the high type. Since $(\beta-1) / \beta \leq \delta$, however, they can sustain cooperation if both of them are revealed to be the low type. In the first period, the low type will select the prescribed action $\pi_{1}^{c}$ at the risk of being exploited by the high type, if $\pi_{1}^{c}$ is small enough to satisfy the second inequality of (17), whereas the high-type player $i$ reveals his own type by selecting $\pi^{i}(1)=0$ if $\pi_{1}^{c}$ is large enough to satisfy the first inequality of (17). It follows from the first inequality of (17) that there always exists a separating equilibrium in which $\pi_{1}^{c}<\bar{\pi}$ provided that $\delta(1-q) \beta /(\beta-1)<1$. In other words, we can always find a separating equilibrium that exhibits gradual cooperation. Furthermore, if $p_{1}$ is so small that

\footnotetext{
${ }^{14}$ Alternatively, we may assume that a player comes to believe that his partner is the high type if he has deviated in any period (not just period 1) without affecting the equilibrium outcome. See also footnote 10.
} 
$p_{1}<(1-\delta)(\beta-1) / \delta(\leq 1$ since $(\beta-1) / \beta \leq \delta)$, the separating equilibrium must exhibit gradualism (if both players are the low type).

There are multiple equilibria, as $\pi_{1}^{c}$ can take any value as long as (17) is satisfied. The high type prefers $\pi_{1}^{c}$ to be high since his expected stage-game payoff in period 1 is $p_{1} \beta \pi_{1}^{c}$ whereas the low type prefers $\pi_{1}^{c}$ to be high if and only if $p_{1}$ is sufficiently high that the chance of being exploited by his partner is small. Indeed, the expected first-period payoff for the low type is $p_{1} \pi_{1}^{c}+\left(1-p_{1}\right)\left[-(\beta-1) \pi_{1}^{c}\right]$, which (weakly) increases with $\pi_{1}^{c}$ if and only if $p_{1} \geq(\beta-1) / \beta$. Thus, if $p_{1} \geq(\beta-1) / \beta$, both types (weakly) prefer $\pi_{1}^{c}$ to be high, so the highest $\pi_{1}^{c}$ that satisfies (17) is to be selected in the efficient equilibrium. If $p_{1}<(\beta-1) / \beta$, on the other hand, the two types have opposite preferences over $\pi_{1}^{c}$, so the efficiency criterion does not select any particular value for $\pi_{1}^{c}$.

It should also be emphasized that the players achieve cooperation if both of them are the low type, even though there is no cooperative pooling equilibrium in this range of parameters. In the pooling equilibrium, the possibility that the other player is the high type cannot be eliminated in the entire course of cooperation, and therefore any cooperation path must satisfy the high type's incentive constraint. In the separating equilibrium, on the other hand, each player eliminates the possibility that his partner is the high type if both players pass the "test" in the first period. Then, the cooperation path need only satisfy the low type's incentive constraint. Whether or not the players can achieve cooperation makes a substantial difference, especially when $p_{1}$ is so large that both players are almost certain that the other player is willing to cooperate.

Now, we turn to the case in which $\delta(1-q)^{2}<(\beta-1) / \beta \leq \delta(1-q)$. A prominent feature of this case is that the players can sustain full cooperation even if one of them is revealed to be the high type. As in the previous case, the cooperation sequence has the property that $\pi_{t}^{c}=\bar{\pi}$ for $t \geq 2$. The cooperation level in the first period $\pi_{1}^{c}$ can be set arbitrarily as long as the following condition is satisfied:

$$
\frac{\left(1-p_{1}\right) \delta(1-q)^{2} \beta \bar{\pi}}{\beta-1} \leq \pi_{1}^{c} \leq \frac{\delta\left(1-p_{1}\right)(1-q) \bar{\pi}}{(\beta-1)[1-\delta(1-q)]} .
$$

To describe the equilibrium strategy, we need to define the set of histories that contain the equilibrium outcome when one of the players is revealed to be the high type. Let $\hat{H}_{t}^{\{i\}}$ denote the set of histories in which any $h_{t} \in \hat{H}_{t}^{\{i\}}$ differs from the unique element of $H_{t}^{\emptyset}$ only in the respect that $\pi^{i}(1)<\pi_{1}^{c}$. In other words, player $i$ has been cooperative except in 
the first period, whereas player $-i$ has been cooperative in every preceding period. ${ }^{15}$ Now, player $i$ 's equilibrium strategy is given by

$$
\begin{aligned}
& \sigma^{i}\left(h_{t}, l\right)=\left\{\begin{array}{cl}
\pi_{t}^{c} & \text { if } h_{t} \in H_{t}^{\emptyset} \cup \hat{H}_{t}^{\{i\}} \cup \hat{H}_{t}^{\{-i\}} \\
0 & \text { otherwise, }
\end{array}\right. \\
& \sigma^{i}\left(h_{t}, h\right)=\left\{\begin{array}{cl}
\pi_{t}^{c} & \text { if } t \geq 2 \text { and } h_{t} \in H_{t}^{\emptyset} \cup \hat{H}_{t}^{\{i\}} \\
0 & \text { otherwise, }
\end{array}\right.
\end{aligned}
$$

while his belief that his partner is the low type is given by

$$
\mu^{i}\left(h_{t}, \theta^{i}\right)=\left\{\begin{array}{cl}
p_{1} & \text { if } h_{t} \in H_{1} \\
0 & \text { if } h_{t} \in H_{t}^{\{-i\}, 1} \cup H_{t}^{I, 1} \\
1 & \text { otherwise }
\end{array}\right.
$$

for any $\theta^{i}$. In equilibrium, the low type chooses $\pi_{1}^{c}$ while the high type chooses 0 in the first period. The players select $\bar{\pi}$ in the second period (unless either one of them come to believe from their first-period actions that both players are the high type), and continue to do so until the end of the game unless a player defects.

Proposition 8 When $\delta(1-q)^{2}<(\beta-1) / \beta \leq \delta(1-q)$, there exists a separating equilibrium in which full cooperation is sustained from the second period onward unless both players are revealed to be the high type through their actions in the first period.

The equilibrium is similar to the one described in Proposition 7, except that in this case where $(\beta-1) / \beta \leq \delta(1-q)$ the players fully cooperate from the second period unless both players are revealed to be the high type. It follows from (18) and $\delta(1-q)^{2} \beta /(\beta-1)<1$ that we can always choose $\pi_{1}^{c}$ smaller than $\bar{\pi}$. Furthermore, if $p_{1}$ is large enough that $1-p_{1}<(\beta-1)[1-\delta(1-q)] / \delta(1-q)(\leq 1$ since $(\beta-1) / \beta \leq \delta(1-q))$, the separating equilibrium must exhibit gradualism (if at least one player is the low type). The proof of Proposition 8 is relegated to the Appendix.

Figure 2 summarizes the equilibrium outcomes in various cases. If outside opportunities do not exist (i.e., if it is common knowledge that neither player has access to the outside option), it is a standard result that players can sustain full cooperation from period 1 and thereafter if and only if $(\beta-1) / \beta \leq \delta$. The possibility that some type of player stochastically

\footnotetext{
${ }^{15}$ Notice that $\hat{H}_{t}^{\{i\}} \subset H_{t}^{\{i\}, 1}$. In any $h_{t} \in H_{t}^{\{i\}, 1} \backslash \hat{H}_{t}^{\{i\}}$, some player has deviated in some period between periods 2 and $t-1$.
} 
obtains the outside option makes cooperation generally more difficult. If $(\beta-1) / \beta>\delta$ or $(\beta-1) / \beta \leq \delta(1-q)^{2}$, the existence of outside options does not change the results. If $\delta(1-q)<(\beta-1) / \beta \leq \delta$, however, the players become unable to cooperate in the pooling equilibrium when outside options are stochastically available. The separating equilibrium generates a better outcome in this case: the players can sustain full cooperation from period 2 if and only if both players are revealed to be the low type (Figure 2 represents this equilibrium by $(l, l))$.

If $\delta(1-q)^{2}<(\beta-1) / \beta \leq \delta(1-q)$, the players gradually increase their cooperation levels in the pooling equilibrium, whereas in the separating equilibrium they can sustain full cooperation from period 2 if and only if at least one player is the low type, which Figure 2 represents by $(l, l),(l, h),(h, l)$. Contrary to the case where $\delta(1-q)<(\beta-1) / \beta \leq \delta$, the low type unambiguously prefers the pooling equilibrium to the separating equilibrium if $(\beta-1) / \beta$ is sufficiently small. To see this claim, let us take an arbitrary $p_{1}>0$. Then, we can find $(\beta-1) / \beta$ that is sufficiently close to $\delta(1-q)^{2}$ so that $p_{1} \geq \hat{p}$. In this case, the players can always sustain full cooperation from period 1 onward in the pooling equilibrium, whereas in the separating equilibrium they can attain full cooperation from period 2 if and only if at least one player is the low type. In addition, the low type would suffer a loss in period 1 if his partner is the high type. The high type also prefers the pooling equilibrium in this case if $p_{1}$ is small enough. Even though $\beta \pi_{1}^{c}$, the high type's stage-game payoff in period 1 when his partner is the low type, may exceed $\bar{\pi}$, the expected stage-game payoff $p_{1} \beta \pi_{1}^{c}$ is small if $p_{1}$ is small. Moreover, the probability that the players cooperate from period 2 in the separating equilibrium, $1-\left(1-p_{1}\right)^{2}$, is small when $p_{1}$ is small.

Proposition 9 Consider the parameter range such that $\delta(1-q)^{2}<(\beta-1) / \beta \leq \delta$. The separating equilibrium is more efficient than the pooling equilibrium if $(\beta-1) / \beta$ is large, whereas the opposite is true if both $(\beta-1) / \beta$ and $p_{1}$ are small.

\section{Concluding Remarks}

We have derived the maximal cooperation processes achieved by two players under asymmetric information on the likelihood of exercising the outside option. If the attractiveness to defect is in the intermediate range, there exist two types of equilibrium: (1) the pooling equilibrium in which players gradually increase the cooperation level as they become more 
confident that their respective partners are not seeking the outside option, and (2) the separating equilibrium in which players start fully cooperating as early as in the second period if they "pass" the first-period "test" on whether or not their respective partners are worth trusting.

As the asymmetry in the likelihood of exercising the outside option increases, cooperation becomes more difficult to sustain, as Bardhan emphasizes, especially for the common pool problem in rural areas. In our incomplete-information game, an increase in the asymmetry expands the parameter range in which the cooperative pooling equilibrium disappears, while the separating equilibrium, in which players sustain cooperation when neither player seeks the outside option, still exists. Thus, we expect that if players can be substantially asymmetric in their attitude toward the outside option, they are likely to judge very quickly whether or not they should continue the relationship.

The pooling equilibrium is expected to arise, on the other hand, if players are within the same, relatively small circle. In most business relationships, the group of active players does not evolve much over time. Personal relationships in schools, workplaces, or local communities are in similar situations. In such an environment, the attractiveness to defect is likely to be small, so the pooling equilibrium tends to be more efficient than the separating equilibrium. Moreover, the initial belief that the other player seeks the outside option is likely to be low, which enables players to start with a relatively high cooperation level in the pooling equilibrium. Since most of the relationships that we observe are ones in relatively small circles, it is not surprising that we commonly observe gradual cooperation with a trust-building stage. 


\section{Appendix}

\section{A Proof of Lemma 5}

What remains to be shown is that $\beta \delta(1-q)\left[p_{t}+\left(1-p_{t}\right)(1-q)\right] /(\beta-1)<1$ for any $p_{t}<\hat{p}$. Since the left-hand side is increasing in $p_{t}$, we need only show $\beta \delta(1-q)[\hat{p}+(1-\hat{p})(1-q)] /(\beta-1)<1$ when $0<\hat{p}<1$. Now, it follows from (9) that

$$
\hat{p}+(1-\hat{p})(1-q)=\frac{\delta(1-q)^{2}+[1-\delta(1-q)]\left[\beta-1-\beta \delta(1-q)^{2}\right]}{\delta(1-q)},
$$

and hence $\beta \delta(1-q)[\hat{p}+(1-\hat{p})(1-q)] /(\beta-1)<1$ if and only if

$$
[\beta-1-\beta \delta(1-q)]\left[\beta-1-\beta \delta(1-q)^{2}\right]<0 .
$$

Since $\delta(1-q)^{2}<(\beta-1) / \beta<\delta(1-q$ ) when $0<\hat{p}<1$ (as is evident from (9)), the first term on the left-hand side of (20) is negative while the second term is positive. Thus, the left-hand side is negative as stated, which implies that the coefficient of $\pi_{t+1}^{c}$ in Lemma 5 is less than 1.

\section{B Proof of Proposition 3}

Since $\pi_{t}^{c}$ never reaches $\bar{\pi}$, the cooperation process is determined by (14) together with $\lim _{t \rightarrow \infty} \pi_{t}^{c}=\bar{\pi}$. Since $\beta \delta(1-q) /(\beta-1)=1$ in this case, (14) can be rewritten as $\pi_{t+1}^{c}=\pi_{t}^{c} / P_{t+1}$. We repeatedly apply this relationship to obtain $\pi_{t+s}^{c}=\pi_{t}^{c} / \prod_{j=1}^{s} P_{t+j}$ for any $s \geq 1$. Consequently, we have

$$
\bar{\pi}=\lim _{s \rightarrow \infty} \pi_{t+s}^{c}=\pi_{t}^{c} / \prod_{j=1}^{\infty} P_{t+j} .
$$

Then, it immediately follows from $p_{t}=\prod_{j=1}^{\infty} P_{t+j}($ derived from $(3))$ that $\pi_{t}^{c}=p_{t} \bar{\pi}$.

\section{Proof of the Claim that $\hat{p}$ increases with $q$}

We show that when $0<\hat{p}<1$, $\hat{p}$ increases with $q$ (i.e., $\partial \hat{p} / \partial q>0$ ). To simplify the computation, we define the function $g(q)$ as the logarithm of $\hat{p}$ :

$$
g(q) \equiv \log \hat{p}
$$




$$
=\log [1-\delta(1-q)]+\log \left[\beta-1-\beta \delta(1-q)^{2}\right]-\log \delta-\log q-\log (1-q)
$$

This function is well-defined since we focus on the case where $\hat{p}>0$. Differentiating $g(q)$ gives us

$$
g^{\prime}(q)=\frac{\delta}{1-\delta(1-q)}+\frac{1}{1-q}+\frac{\beta \delta\left(1-q^{2}\right)-(\beta-1)}{q\left[\beta-1-\beta \delta(1-q)^{2}\right]} .
$$

The first two terms on the right-hand side are unambiguously positive. Both the numerator and denominator of the third term are also positive as

$$
\beta \delta\left(1-q^{2}\right)-(\beta-1)>\beta\left[\delta(1-q)-\frac{\beta-1}{\beta}\right]>0
$$

when $\hat{p}<1$, and

$$
\beta-1-\beta \delta(1-q)^{2}=\beta\left[\frac{\beta-1}{\beta}-\delta(1-q)^{2}\right]>0
$$

when $\hat{p}>0$. Thus, we find that $g^{\prime}(q)>0$ for any $q \in(0,1)$, and hence $\hat{p}$ increases with $q$.

\section{Proof of Proposition 7}

It is straightforward that the belief system is consistent with the strategy profile. Now, let us consider player $i$ 's incentive to follow the prescribed strategy from period 2. As long as both players have been acting cooperatively (i.e., $h_{t} \in H_{t}^{\emptyset}$ ), they both believe that their respective partners are the low type. The low type's payoff is $\bar{\pi} /(1-\delta)$ if he follows the prescribed strategy and $\beta \bar{\pi}$ if he optimally deviates, given that his partner is also the low type. He follows the strategy since $\bar{\pi} /(1-\delta) \geq \beta \bar{\pi}$ holds when $(\beta-1) / \beta \leq \delta$. If either player had deviated, i.e., $h_{t} \notin H_{t}^{\emptyset}$, the low type expects that his partner continues to select 0 . Thus, it is rational for him to choose his best response $\pi^{i}(t)=0$.

The high type, on the other hand, selects 0 in any occasion from period 2. Obviously, this selection is rational if either player has deviated (i.e., $h_{t} \notin H_{t}^{\emptyset}$ ), since he expects that his partner selects 0 in every following period. If $h_{t} \in H_{t}^{\emptyset}$, he obtains $\beta \bar{\pi}$ if he follows the prescribed strategy and $\bar{\pi}+\delta(1-q) \beta \bar{\pi}$ if he delays his deviation for one period. Since $(\beta-1) / \beta>\delta(1-q)$ implies that $\beta \bar{\pi}>\bar{\pi}+\delta(1-q) \beta \bar{\pi}$, it is rational for him to set 0 in the current period $t$.

In period 1 , the low type selects $\pi_{1}^{c}$ if

$$
p_{1}\left[\pi_{1}^{c}+\frac{\delta \bar{\pi}}{1-\delta}\right]-\left(1-p_{1}\right)(\beta-1) \pi_{1}^{c} \geq p_{1} \beta \pi_{1}^{c}
$$




$$
\pi_{1}^{c} \leq \frac{p_{1} \delta \bar{\pi}}{(1-\delta)(\beta-1)}
$$

The left-hand side of the first inequality represents the equilibrium payoff while the righthand side represents the payoff when he deviates by setting his first period cooperation level at 0 . It follows from $(22)$ that $\pi_{1}^{c}$ must be smaller than $\bar{\pi}$ if $p_{1}<(1-\delta)(\beta-1) / \delta$. (Notice that $(1-\delta)(\beta-1) / \delta \leq 1$ when $(\beta-1) / \beta \leq \delta$.) Similarly, the high type selects his cooperation level at 0 in period 1 if

$$
\begin{aligned}
p_{1} \beta \pi_{1}^{c} & \geq p_{1}\left[\pi_{1}^{c}+\delta(1-q) \beta \bar{\pi}\right]-\left(1-p_{1}\right)(\beta-1) \pi_{1}^{c} \\
\pi_{1}^{c} & \geq \frac{p_{1} \delta(1-q) \beta \bar{\pi}}{\beta-1} .
\end{aligned}
$$

For both (22) and (23) to hold simultaneously, it is necessary that $p_{1} \delta(1-q) \beta \bar{\pi} /(\beta-1)<$ $p_{1} \delta \bar{\pi} /(1-\delta)(\beta-1)$, which can be reduced to $(\beta-1) / \beta<q+\delta(1-q)$. Since this inequality always holds, there exists $\pi_{1}^{c}$ that simultaneously satisfies (22) and (23). If $\pi_{1}^{c}$ is so chosen, the prescribed strategy profile and belief system constitute a perfect Bayesian equilibrium as we have shown.

\section{E Proof of Proposition 8}

As in the previous case, it is straightforward that the belief system is consistent. Now, we consider player $i$ 's incentive to follow the prescribed strategy for $t \geq 2$. From period 2 , both players follow the grim-trigger-strategy if both players believe that at least one of them is the low type. Since the high type's incentive constraint is tighter, we need only show that the high type's incentive constraint is satisfied when his partner is revealed to be the low type and both players have been acting cooperatively since period 2 . In this case, he obtains $\bar{\pi} /[1-\delta(1-q)]$ if he follows the prescribed strategy and $\beta \bar{\pi}$ if he optimally deviates. Since $\bar{\pi} /[1-\delta(1-q)] \geq \beta \bar{\pi}$ when $(\beta-1) / \beta \leq \delta(1-q)$, he selects $\bar{\pi}$ following the prescribed strategy.

We have thus shown that $\sigma^{i}\left(h_{t}, l\right)=\pi_{t}^{c}$ if $h_{t} \in H_{t}^{\emptyset} \cup \hat{H}_{t}^{\{i\}} \cup \hat{H}_{t}^{\{-i\}}$, and $\sigma^{i}\left(h_{t}, h\right)=\pi_{t}^{c}$ if $t \geq 2$ and $h_{t} \in H_{t}^{\emptyset} \cup \hat{H}_{t}^{\{i\}}$.

It is obvious that player $i$, irrespective of his type, selects 0 if both players have deviated in the first period or either player has deviated in a previous period other than the first period, since in these cases his partner is supposed to select 0 from the current period onward. It is also rational for the high type to select 0 in the case where he believes that his partner is the high type while his partner believes mistakenly that he is the low type (and hence both 
have been acting cooperatively). In such a case, the high type obtains $\beta \bar{\pi}$ if he follows his prescribed strategy, and $\bar{\pi}+\delta(1-q)^{2} \beta \bar{\pi}$ if he delays revealing his type for one period. The former value is greater than the latter when $\delta(1-q)^{2} \leq(\beta-1) / \beta$.

In period 1 , the low type selects $\pi_{1}^{c}$ if

$$
p_{1}\left[\pi_{1}^{c}+\frac{\delta}{1-\delta} \bar{\pi}\right]+\left(1-p_{1}\right)\left[(1-\beta) \pi_{1}^{c}+\frac{\delta(1-q)}{1-\delta(1-q)} \bar{\pi}\right] \geq p_{1}\left[\beta \pi_{1}^{c}+\frac{\delta}{1-\delta} \bar{\pi}\right] .
$$

He has an incentive to choose $\pi_{1}^{c}$ rather than 0 since a deviation eliminates the possibility of cooperation from the next period onward in the case where his partner is the high type. The above inequality can be rewritten as

$$
\pi_{1}^{c} \leq \frac{\delta\left(1-p_{1}\right)(1-q) \bar{\pi}}{(\beta-1)[1-\delta(1-q)]}
$$

On the other hand, the high type selects his equilibrium action 0 in period 1 if $\pi_{1}^{c}$ is large enough that

$$
\begin{aligned}
p_{1}\left[\beta \pi_{1}^{c}+\frac{\delta(1-q)}{1-\delta(1-q)} \bar{\pi}\right] & \geq p_{1}\left[\pi_{1}^{c}+\frac{\delta(1-q)}{1-\delta(1-q)} \bar{\pi}\right]+\left(1-p_{1}\right)\left[(1-\beta) \pi_{1}^{c}+\delta(1-q)^{2} \beta \bar{\pi}\right] \\
\pi_{1}^{c} & \geq \frac{\left(1-p_{1}\right) \delta(1-q)^{2} \beta \bar{\pi}}{\beta-1} .
\end{aligned}
$$

Notice that the second term on the right-hand side of the first inequality represents the payoff when he chooses $\pi_{1}^{c}$ in period 1 and his partner is the high type. In this case, he loses $(\beta-1) \pi_{1}^{c}$ in period 1 , but he obtains $\beta \bar{\pi}$ in period 2 since his partner selects $\bar{\pi}$ in period 2 , believing mistakenly that player $i$ is the low type.

It is necessary for both (24) and (25) to hold simultaneously that

$$
\frac{\left(1-p_{1}\right) \delta(1-q)^{2} \beta \bar{\pi}}{\beta-1} \leq \frac{\delta\left(1-p_{1}\right)(1-q) \bar{\pi}}{(\beta-1)[1-\delta(1-q)]},
$$

which is reduced to $(\beta-1) / \beta \leq \delta(1-q)^{2}+q$. Since $(\beta-1) / \beta<\delta(1-q)$ and $\delta(1-q)^{2}+q=$ $(1-q) \delta(1-q)+q>\delta(1-q)$, we find that this inequality always holds. Thus, we can choose $\pi_{1}^{c}$ so as to satisfy (18) to make the prescribed strategy profile and belief system constitute a perfect Bayesian equilibrium. 


\section{References}

Admati, A.R., Perry, M., 1991. Joint projects without commitment. Review of Economic Studies 58, 259-276.

Andreoni, J., Samuelson, L., 2006. Building rational cooperation. Journal of Economic Theory 127, 117-154.

Bardhan, P., 2004. Scarcity, Conflicts, and Cooperation: Essays in the Political and Institutional Economics of Development. Cambridge: MIT Press.

Blonski, M., Probst, D.A., 2004. The emergence of trust. Unpublished manuscript, Goethe University, Frankfurt am Main.

Compte, O., Jehiel, P., 2004. Gradualism in bargaining and contribution games. Review of Economic Studies 71, 975-1000.

Datta, S., 1993. Building trust. Unpublished manuscript.

Fershtman, C., Gandal, N., 1994. Disadvantageous semicollusion. International Journal of Industrial Organization 12, 141-154.

Fudenberg, D., Maskin, E., 1986. The folk theorem in repeated games with discounting or with incomplete information. Econometrica 54, 533-554.

Fujiwara-Greve, T., 2001. On voluntary and repeatable partnerships under no information flow. Unpublished manuscript, Keio University.

Furusawa, T., Kawakami, T., 2006. Gradual cooperation in the existence of outside options. COE/RES Discussion Paper Series, No. 180, Hitotsubashi University.

Furusawa, T., Lai, E., 1999. Adjustment costs and gradual trade liberalization. Journal of International Economics 49, 333-361.

Ghosh, P., Ray, D., 1996. Cooperation in community interaction without information flows. Review of Economic Studies 63, 491-519.

Kranton, R.E., 1996. The formation of cooperative relationships. Journal of Law, Economics, and Organization 12, 214-233.

Lindsey, J., Polak, B., Zeckhauser, R., 2003. Free love, fragile fidelity, and forgiveness: rival social conventions under hidden information. Unpublished manuscript, Yale University.

Lockwood, B., Thomas, J.P., 2002. Gradualism and irreversibility. Review of Economic Studies 69, 339-356. 
Martin, S., 1995. R\&D joint ventures and tacit product market collusion. European Journal of Political Economy 11, 733-741.

Marx, L.M., Matthews., S.A., 2000. Dynamic voluntary contribution to a public project. Review of Economic Studies 67, 327-358.

Matsushima, H., 1990. Long-term partnership in a repeated prisoner's dilemma with random matching. Economics Letters 34, 245-248.

Petersen, M.A., Rajan, R.G., 1994. The benefits of lending relationships: evidence from small business data. Journal of Finance 49, 3-37.

Rob, R., Yang, H., 2006. Long-term relationships as safeguards. Unpublished manuscript, Ohio State University.

Roth, D., 1996. A theory of partnership dynamics. Games and Economic Behavior 12, 95-112.

Sobel, J., 1985. A theory of credibility. Review of Economic Studies 52, 557-573.

Watson, J., 1999. Starting small and renegotiation. Journal of Economic Theory 85, 52-90.

Watson, J., 2002. Starting small and commitment. Games and Economic Behavior 38, 176-199. 


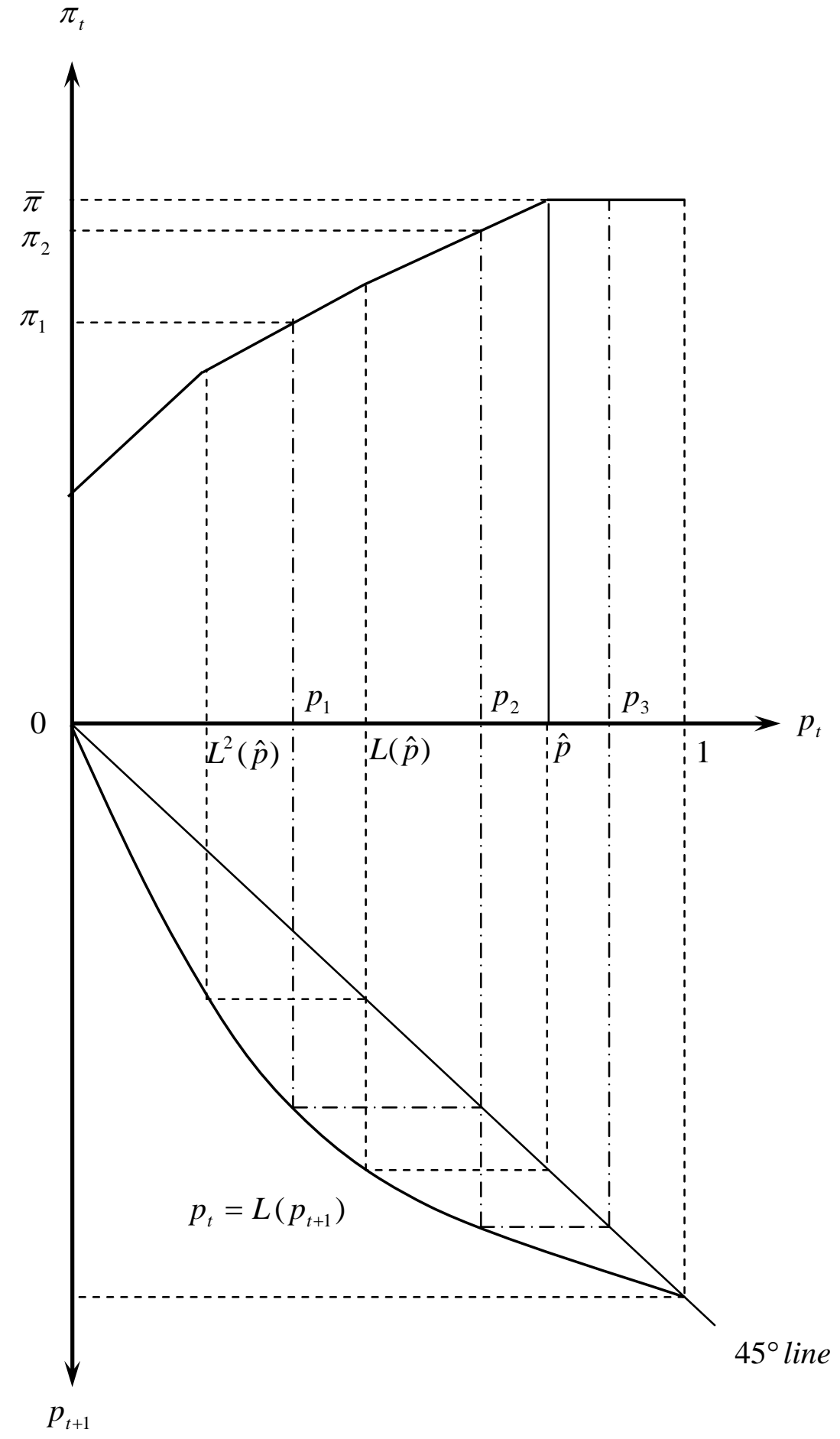

Figure 1. The Efficient Cooperation Process 


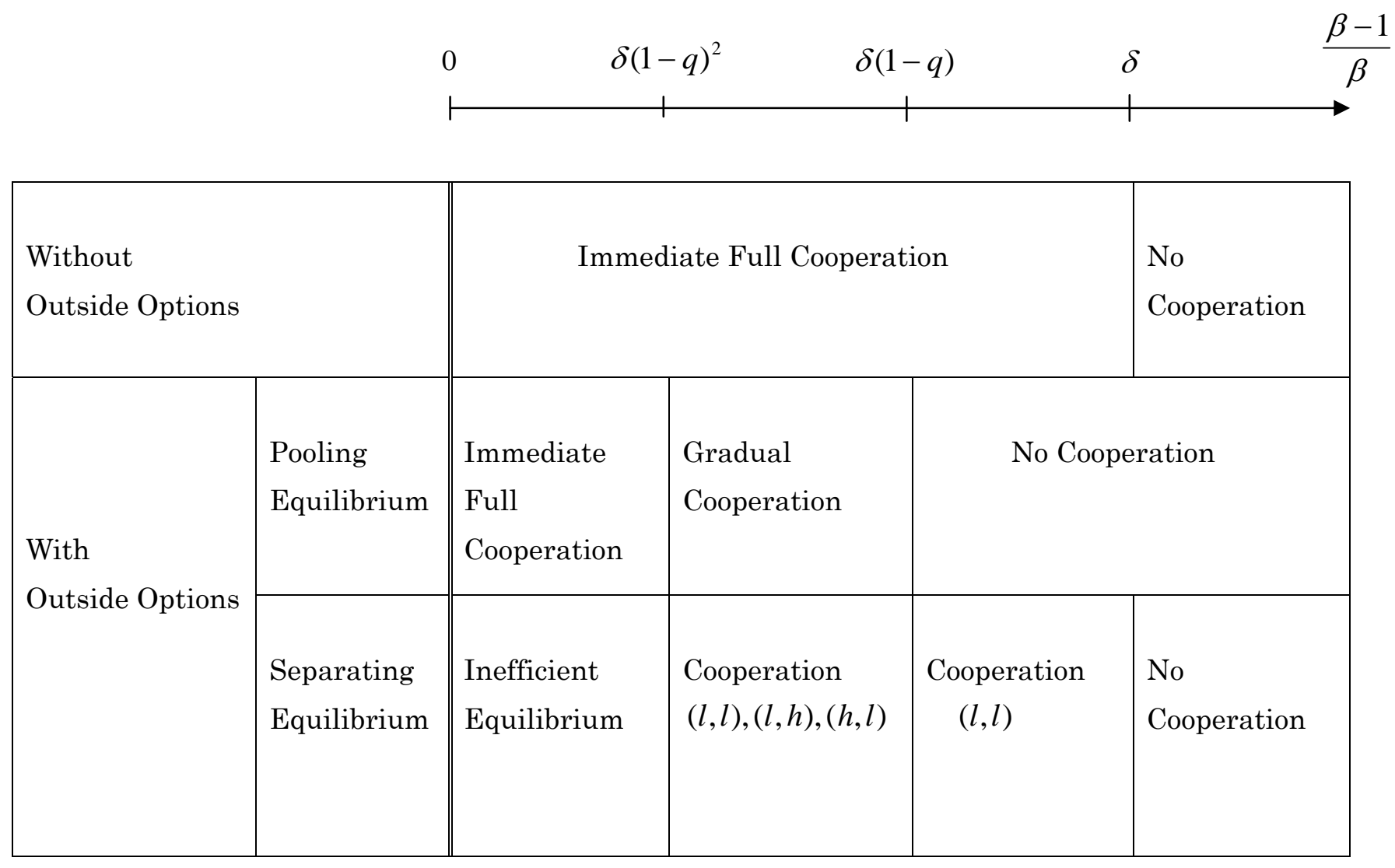

Figure 2. Summary of the Result 